\title{
A Novel Type 2 Diabetes Mouse Model of Combined Diabetic Kidney Disease and Atherosclerosis
}

\author{
Karin E. Bornfeldt, ${ }^{\dagger \dagger}$ Farah Kramer, ${ }^{*}$ Anna Batorsky, ${ }^{\dagger}$ Jinkuk Choi, ${ }^{\ddagger \S \pi}$ Kelly L. Hudkins, ${ }^{\dagger}$ Peter Tontonoz, ${ }^{\ddagger \delta 币}$ Charles E. Alpers, \\ and Jenny E. Kanter* \\ From the Departments of Medicine* and Pathology, ${ }^{\dagger}$ UW Medicine Diabetes Institute, University of Washington School of Medicine, Seattle, Washington; and \\ the Departments of Pathology ${ }^{\ddagger}$ and Laboratory Medicine ${ }^{\S}$ and the Molecular Biology Institute, "University of California, Los Angeles, California
}

\author{
Accepted for publication \\ October 19, 2017. \\ Address correspondence to \\ Jenny E. Kanter, Ph.D., UW \\ Medicine Diabetes Institute, \\ University of Washington \\ School of Medicine, Seattle, \\ WA 98109. E-mail: jenka@ \\ uw.edu.
}

\begin{abstract}
Diabetic kidney disease and atherosclerotic disease are major causes of morbidity and mortality associated with type 2 diabetes (T2D), and diabetic kidney disease is a major cardiovascular risk factor. The black and tan, brachyury (BTBR) mouse strain with leptin deficiency $\left(\right.$ Lep $\left.^{o b}\right)$ has emerged as one of the best models of human diabetic kidney disease. However, no T2D mouse model of combined diabetic kidney disease and atherosclerosis exists. Our goal was to generate such a model. To this end, the lowdensity lipoprotein ( $L D L)$ receptor was targeted for degradation via inducible degrader of the $L D L$ receptor (IDOL) overexpression, using liver-targeted adenoassociated virus serotype DJ/8 (AAV-DJ/8) in BTBR wild-type and BTBR Lep ${ }^{o b}$ mice. Liver-targeted IDOL-AAV-DJ/8 increased plasma LDL cholesterol compared with the control enhanced green fluorescent protein AAV-DJ/8. IDOL-induced dyslipidemia caused formation of atherosclerotic lesions of an intermediate stage, which contained both macrophages and smooth muscle cells. BTBR Lep ${ }^{o b}$ mice exhibited diabetic kidney disease. IDOL-induced dyslipidemia worsened albuminuria and glomerular macrophage accumulation but had no effect on mesangial expansion or podocyte numbers. Thus, by inducing hepatic degradation of the LDL receptor, we generated a T2D model of combined kidney disease and atherosclerosis. This model provides a new tool to study mechanisms, interactions, and treatment strategies of kidney disease and atherosclerosis in T2D. (Am J Pathol 2018, 188: 343-352; https://doi.org/10.1016/j.ajpath.2017.10.012)
\end{abstract}

Microvascular and macrovascular complications, such as kidney disease and atherosclerotic disease, are major causes of morbidity and mortality associated with type 2 diabetes (T2D). Diabetes results in a doubling of cardiovascular risk, ${ }^{1}$ but the mechanisms whereby diabetes accelerates the underlying process of atherosclerosis are not fully understood. In addition to cardiovascular complications, diabetes is the leading cause of chronic kidney disease and renal failure, and at least $35 \%$ of adults with T2D have diabetic kidney disease (DKD), defined as albuminuria or an impaired estimated glomerular filtration rate. ${ }^{2}$ The presence of kidney disease also increases the risk of cardiovascular events in patients with and without diabetes ${ }^{3,4}$; however, the interactions between DKD and atherosclerosis are not clear, partly because few animal models that accurately reflect human DKD exist. Although many mouse models of atherosclerosis are available, few are models of T2D-acclerated atherosclerosis, and no model also exhibits DKD. The black and tan, brachyury (BTBR) mouse strain with leptin deficiency [Lep ${ }^{o b}$ (OB)] has emerged as one of the best models of human DKD because in addition to exacerbated albuminuria it features morphologic characteristics of DKD, such as increased mesangial matrix deposition and sclerosis, mesangiolysis,

\footnotetext{
Supported by NORC Pilot and Feasibility grant P30 DK035816 (J.E.K.) and in part by American Diabetes Association grant 1-16-IBS-15 (J.E.K.), Diabetes Research Center Pilot and Feasibility grant P30 DK017047 (J.E.K.), NIH grants R01HL062887 (K.E.B.), P01HL092969 (K.E.B.), R01HL126028 (K.E.B.), R01HL066088 (P.T.), and DK08339 (C.E.A. and K.L.H.). The adenoassociated viruses were produced through grant P30 DK017047 by the Diabetes Research Center's Vector and Transgenic Mouse core at the University of Washington.

Disclosures: None declared.
} 
podocyte loss, and macrophage accumulation. ${ }^{5}$ However, BTBR mice do not develop significant atherosclerosis ${ }^{5}$ because of the low levels of low-density lipoprotein (LDL) compared with high-density lipoprotein (HDL). The traditional strategy to render mouse models susceptible to atherogenesis is to reduce clearance of LDL via timeconsuming and costly breeding to mice deficient in apolipoprotein $\mathrm{E}$ or the LDL receptor $\left(\mathrm{Ldlr}^{-/-}\right.$mice). This results in a more human-like lipoprotein profile and increased LDL levels compared with HDL levels. ${ }^{6-8}$ Recently, novel pathways that reduce LDL receptor levels and thus increase LDL cholesterol (LDL-C) and total cholesterol levels have been discovered. One such pathway involves the E3 ubiquitin ligase inducible-degrader of the LDL receptor (IDOL). ${ }^{9,10}$ IDOL targets the LDL receptor for ubiquitin-mediated lysosomal degradation, and IDOL overexpression using an adenoassociated viral (AAV) approach increases LDL-C in wild-type (WT) C57Bl/6 mice. ${ }^{9,11}$ Furthermore, transgenic overexpression of human IDOL in WT C57B1/6 mice results in lipoprotein profiles similar to those of $\mathrm{Ldlr}^{-1-}$ mice and results in concomitant atherosclerosis. ${ }^{12}$

There is a strong association between suboptimal glycemic control and DKD; however, despite current antiglycemic therapies, diabetes can still be attributed to almost half of all end-stage renal disease cases. ${ }^{2}$ Dyslipidemia, especially diabetic dyslipidemia with elevated triglyceride and low HDL cholesterol (HDL-C) levels, is an independent risk factor for progression of renal disease in individuals with T2D. ${ }^{13}$

Our goal was twofold; to generate a much needed T2D mouse model of combined DKD and atherosclerosis and to investigate whether dyslipidemia worsens DKD. The LDL receptor was targeted for degradation by liver-specific AAV serotype DJ/8 (AAV-DJ/8)-mediated overexpression of IDOL in BTBR WT and OB mice. We demonstrate generation of a T2D mouse model of combined DKD and atherosclerosis and show that IDOL-mediated dyslipidemia worsens diabetic albuminuria and glomerular macrophage accumulation.

\section{Materials and Methods}

\section{Liver-Specific AAV-DJ/8}

The AAV serotype DJ/8 exhibits high infectivity in vivo, ${ }^{14}$ and is not known to cause disease or immune reactions. An AAV-DJ/8 encoding a degradation resistant mutant (K293R, K309R, K310R, K320R) IDOL,${ }^{10,12}$ with bicistronic expression of enhanced green fluorescent protein (eGFP) under control of the liver-specific human thyroxinebinding globulin promoter was generated. The same AAV-DJ/8 that expressed liver-specific eGFP only was used as a control.

\section{Mice}

All animal work was approved by the Institutional Animal Care and Use Committee at the University of Washington.
Four- to 5-week-old male BTBR WT or OB mice were injected retro-orbitally with $1 \times 10^{11}$ viral genomes of AAV-DJ/8 containing eGFP or the human IDOL mutant. To increase uniformity of the study groups, only male mice were chosen for the study. Animals were randomized into each experimental group based on similar body weights with a target of 12 to 15 per group (statistically predetermined, based on power calculations). The viral dose was based on a pilot experiment. After the AAV injection, the animals were fed a semipurified, high-fat, cholesterolcontaining diet (40\% of calories from fat, $1.25 \%$ added cholesterol; TD 00244, Enivgo, Madison, WI) ${ }^{15}$ for 18 weeks. BTBR OB mice exhibit increased mortality after 24 weeks of age, preventing us from performing studies past this age. ${ }^{5}$ To verify the effect of IDOL-induced dyslipidemia in the $\mathrm{C} 57 \mathrm{~B} 1 / 6$ strain in which atherosclerosis is traditionally studied, WT and OB C57B1/6 mice were injected with IDOL-AAV-DJ/8 and treated the same way as the BTBR mice. The high-fat diet used is not an obesogenic diet because WT C57B1/6 mice had gained approximately 30\% body weight after 17 weeks on diet. Body weights were monitored weekly, and glucose and plasma cholesterol were measured after 2, 8, and 18 weeks of diet. Lipid and glucose measurements were performed on ad libitum fed animals.

\section{Albuminuria, Plasma Cystatin C, and Blood Urea Nitrogen}

Urine was collected during a 4- to 6-hour fast in the last week of the study in specialized cages, in which the urine was collected into the bottom portion of the cage through a straining mesh. Urinary albumin was measured using a mouse albumin enzyme-linked immunosorbent assay and normalized to urinary creatinine levels (Albuwell, and Creatinine Companion, Exocell, Philadelphia, PA). Urinary volumes and total 24-hour urine and albumin secretion were also calculated (Supplemental Table S1). Plasma cystatin C was measured using a cystatin C enzyme-linked immunosorbent assay (R\&D Systems via Fisher Scientific, Hampton, $\mathrm{NH}$ ), and plasma urea was measured using a Bioassay Systems Urea assay kit (Fisher Scientific). Blood urea nitrogen was calculated according to the manufacturer's instructions.

\section{Lipid Measurements}

Plasma triglycerides, nonesterified fatty acids, and cholesterol were measured using colorimetric assays from SigmaAldrich (St Louis, MO) and Wako Chemicals (Richmond, VA), respectively. Cholesterol lipoprotein profiles were determined as previously described ${ }^{16}$ and very low-density lipoprotein-C, LDL-C, and HDL-C were calculated from these. Cortex triglycerides were measured using the same colorimetric assay (Sigma-Aldrich) and normalized to milligrams of protein in the extracted tissue. 
Table 1 List of Primers Used in This Study and Their Sources

\begin{tabular}{|c|c|c|}
\hline Gene & Primer sequences & Source \\
\hline Mylip (mouse and human) & $\begin{array}{l}\text { F 5'-CATCTTACAGGAGCAGACTAGGC-3' } \\
\text { R 5'-TTGGCAGTGTTCTGGTTGTAG-3' }\end{array}$ & Invitrogen* \\
\hline MYLIP (human specific) & $\begin{array}{l}\text { F 5'-AAACCTGAGAAACCGGATCTC-3' } \\
\text { R 5'-GCTCCACGAAGAACTTGACTCTA-3' }\end{array}$ & Invitrogen \\
\hline Rn18s & $\begin{array}{l}\text { F 5'-CATTAAATCAGTTATGGTTCCTTTGG-3' } \\
\text { R 5'-CCCGTCGGCATGTATTAGCT-3' }\end{array}$ & Invitrogen \\
\hline Vcam1 & $\begin{array}{l}\text { F 5'-TGCACAGTCCCTAATGTGTATCC-3' } \\
\text { R 5'-GACTTTATGCCCATTTCCTCCA-3' }\end{array}$ & Invitrogen \\
\hline Icam 1 & $\begin{array}{l}\text { F 5'-GTGATGCTCAGGTATCCATCCA-3' } \\
\text { R 5'-CACAGTTCTCAAAGCACAGCG-3' }\end{array}$ & Invitrogen \\
\hline Il1b & $\begin{array}{l}\text { F 5'-GGGCTGCTTCCAAACCTTTG-3' } \\
\text { R 5'-TGATACTGCCTGCCTGAAGCTC-3' }\end{array}$ & Invitrogen \\
\hline Emr1 & $\begin{array}{l}\text { F 5'-TGACTCACCTTGTGGTCCTAA-3' } \\
\text { R 5'-CTTCCCAGAATCCAGTCTTTCC-3' }\end{array}$ & Invitrogen \\
\hline Acox 1 & $\begin{array}{l}\text { F 5'-GAGCAGCAGGAGCGTTTCTT-3' } \\
\text { R 5'-CAGGACTATCGCATGATTGGAAG-3' }\end{array}$ & Invitrogen \\
\hline Ppara & $\begin{array}{l}\text { F 5'-TTTCGGCGAACTATTCGGCTG-3' } \\
\text { R 5'-GGCATTTGTTCCGGTTCTTCTT-3' }\end{array}$ & Invitrogen \\
\hline Ppargc1a & $\begin{array}{l}\text { F 5'-AAGTGGTGTAGCGACCAATCG-3' } \\
\text { R 5'-AATGAGGGCAATCCGTCTTCA-3' }\end{array}$ & Invitrogen \\
\hline
\end{tabular}

*Carlsbad, CA

$F$, forward; $R$, reverse.

\section{Analysis of Atherosclerosis and Kidney Histologic Analysis}

At the end of the study, the mice were euthanized, blood was collected, and the animals were perfused gently with phosphate-buffered saline through the apex of the heart. The aorta, heart, brachiocephalic artery, liver, and kidneys were dissected out and placed in $10 \%$ neutral buffered formalin (Sigma-Aldrich). Another piece of liver was snap frozen for mRNA and protein analysis. The aortas (from the heart to the iliac bifurcation) were opened longitudinally, and atherosclerosis was determined en face after Sudan IV staining, as previously described. ${ }^{15}$ The aortic sinus and a subset of aortas were sectioned, stained with Movat's pentachrome stain, and analyzed for lesion morphologic features. ${ }^{15,17}$ The kidneys were cut longitudinally, embedded in paraffin, and sectioned. A subset of kidneys were embedded in optimal cutting temperature embedding media, sectioned, and stained for neutral lipid deposition using Oil Red O. For ultrastructural analysis, kidneys were fixed in $10 \%$ neutral buffered formalin, postfixed in $1 \%$ osmium tetroxide, then processed and embedded in Eponate 12 resin using routine protocols. Approximately $0.1-\mu \mathrm{m}$ sections were stained with uranyl acetate and lead citrate and examined using a Jeol JEM-1230 electron microscope. For analysis of RNA, the cortex was separated from the medulla and snap frozen. Periodic acid-Schiff (PAS) and silver methenamine stains were used to quantify mesangial expansion. ${ }^{5}$ Macrophage accumulation in the aorta and glomeruli was assessed using galectin-3 (Mac-2) immunostaining, which was performed after sodium citrate/ Tween antigen retrieval (monoclonal rat anti-mouse Mac-2 antibody: CL8942AP at $1 \mu \mathrm{g} / \mathrm{mL}$; Cedarlane, Burlington, $\mathrm{NC})$. For quantification of glomerular changes, 15 to 20 glomeruli were randomly photographed and the degree of glomerular matrix accumulation was quantified by computer image analysis [ImagePro Plus image analysis software version 7 (Media Cybernetics, Rockville, MD)], as previously described. ${ }^{5}$ Podocyte numbers were estimated based on a method described by Venkatareddy et al. ${ }^{18}$ Briefly, podocytes were identified using a p57 stain (SC8298 at $0.2 \mu \mathrm{g} / \mathrm{mL}$; Santa Cruz Biotechnology, Dallas, TX), and glomerular podocytes were counted and their diameters measured to estimate the total number of podocytes per glomerulus. Mac2 -positive macrophages within glomeruli were counted in a minimum of 10 glomerular cross sections and expressed as the mean number of cells per glomerulus. For all stains, a negative control antibody of the same type and concentration was used. All histologic analyses were performed in a masked fashion.

Western Blot Analysis and Real-Time Quantitative PCR

For Western blot analysis, proteins were separated via SDS-PAGE and transferred to polyvinylidene difluoride 
A

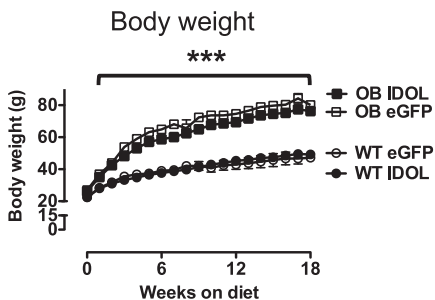

D

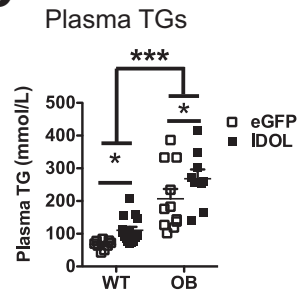

G

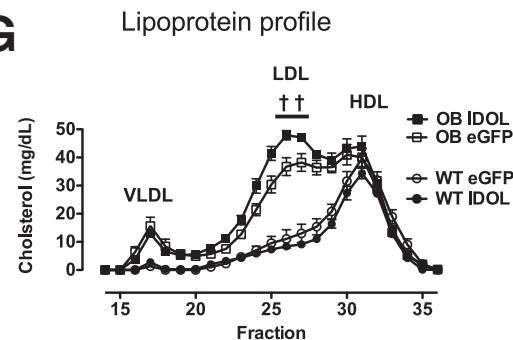

B

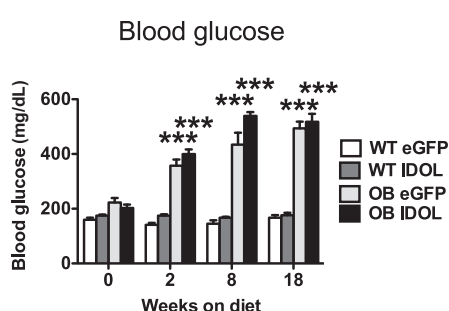

E

Liver MYLIP mRNA

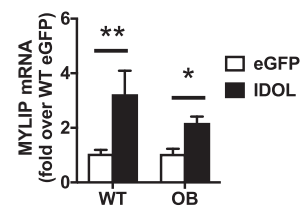

C

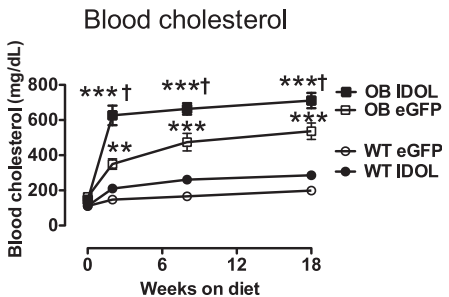

F

Liver LDL receptor

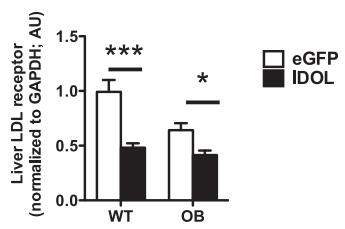

H

\section{Lipoproteins}

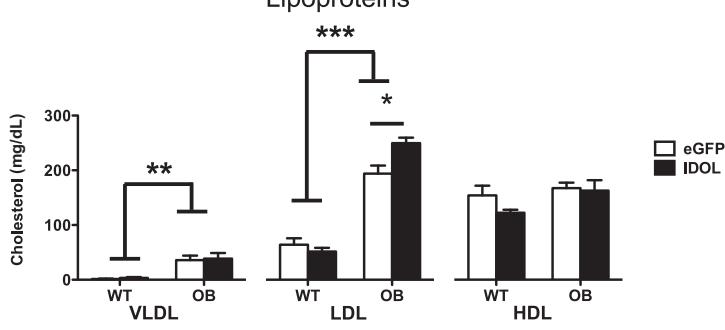

Figure 1 Inducible degrader of the low-density lipoprotein (LDL) receptor (IDOL) overexpression results in elevated cholesterol levels. Human degradationresistant IDOL was expressed using a liver-specific adenoassociated virus (AAV) serotype DJ/8 [or enhanced green fluorescent protein (eGFP)-AAV-DJ/8 as a control) in 5-week-old male black and tan, brachyury (BTBR) wild-type (WT) and BTBR leptin-deficient (OB) mice. The mice were then fed a high-fat diet for 18 weeks. A: Body weight throughout the study. B: Blood glucose. C: Blood cholesterol. D: Plasma triglycerides (TG). E: Liver MYLIP mRNA using primers that detect both mouse and human MYLIP (gene encoding IDOL). F: Liver LDL receptor levels determined by Western blot and normalized to glyceraldehyde-3-phosphate dehydrogenase. G: Cholesterol lipoprotein profiles. H: Quantification of cholesterol in each lipoprotein based on G. Data are expressed as means \pm SEM. $n=9$ to $15(\mathbf{A}-\mathbf{D}, \mathbf{H}) ; n=5$ to $9(\mathbf{E}) ; n=4$ to $7(\mathbf{F}) ; n=3$ to $4(\mathbf{G}) .{ }^{*} P<0.05,{ }^{*} P P<0.01$, and ${ }^{*} * * P<0.001$ compared with the WT eGFP group; ${ }^{\dagger} P<0.05$ compared with the OB eGFP group (two-way analysis of variance). HDL, high-density lipoprotein; VLDL, very low-density lipoprotein.

membranes followed by incubation with the following antibodies: LDL receptor (goat polyclonal, AF2255 at $0.2 \mu \mathrm{g} / \mathrm{mL}$; R\&D Systems, Minneapolis, MN), glyceraldehyde3-phosphate dehydrogenase (SC24778 at $0.2 \mu \mathrm{g} / \mathrm{mL}$; Santa Cruz Biotechnology). Total RNA was isolated using Qiagen RNeasy Mini Kits. To remove trace genomic DNA, all samples were DNase treated. Real-time quantitative PCR was run using SYBR Green PCR Master Mix (Fermentas via Thermo Fisher Scientific, Waltham, MA), as described previously. ${ }^{19}$ After each assay, a dissociation curve was run to confirm specificity of all PCR amplicons. All primer reactions were also analyzed on agarose gels for correct size and the presence of a single reaction product. Resulting $\mathrm{Ct}$ values were normalized to $R n 18 s$, and the $\Delta \Delta \mathrm{Ct}$ method was then used to express values as fold-over control samples. All samples were run in at least duplicates, and statistical analysis was performed on $2^{-(\Delta \mathrm{Ct})}$ values. Primer sequences used are given in Table 1.

\section{Statistical Analysis}

All data are expressed as means \pm SEM. Statistical analysis was performed using two-tailed unpaired $t$-test or one or two-way analysis of variance with Tukey's multiple comparison post hoc test for normally distributed data or Bonferroni multiple comparisons. Statistical outliers (Grubb's test) were excluded. $P<0.05$ was considered statistically significant.

\section{Results}

IDOL Overexpression Results in Elevated Cholesterol Levels

To target degradation of the LDL receptor in the liver, AAV-DJ/8 that expressed a human degradation-resistant IDOL mutant under control of a liver-specific promoter ${ }^{10,11}$ was injected into 4- to 5-week-old male WT and OB mice on the BTBR background. An AAV that expressed eGFP was used as control. After AAV injections, mice were fed a semipurified, high-fat, cholesterol-containing diet to promote atherosclerosis. ${ }^{15}$ As expected, OB mice rapidly became obese, gaining more than $200 \%$ body weight during 18 weeks (weight gain was $210 \% \pm 22 \%$ in eGFP OB mice and $211 \% \pm 19 \%$ in IDOL OB mice). After 18 weeks, the BTBR OB mice weighed a mean of $25 \mathrm{~g}$ more than the BTBR WT controls (Figure 1A). Injection of IDOL-AAV 
A

\section{Albuminuria}

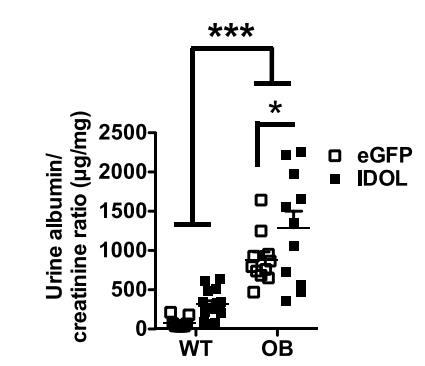

C
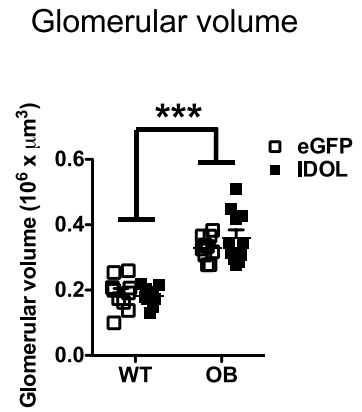

B
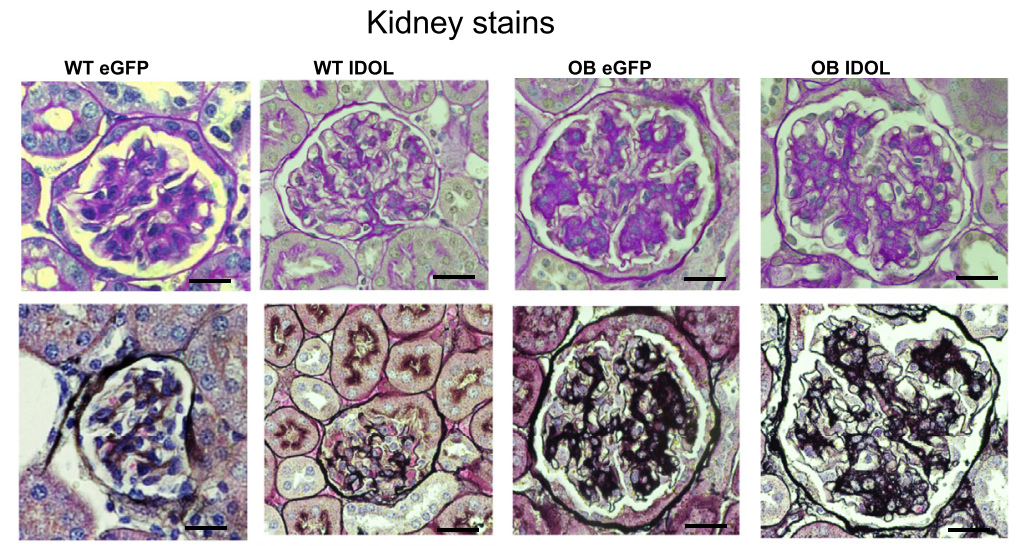

PAS
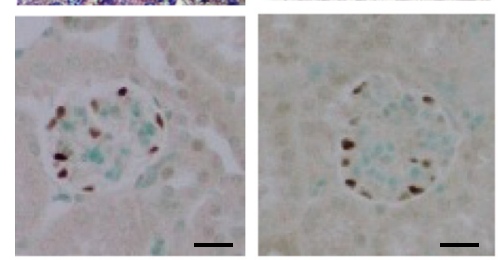
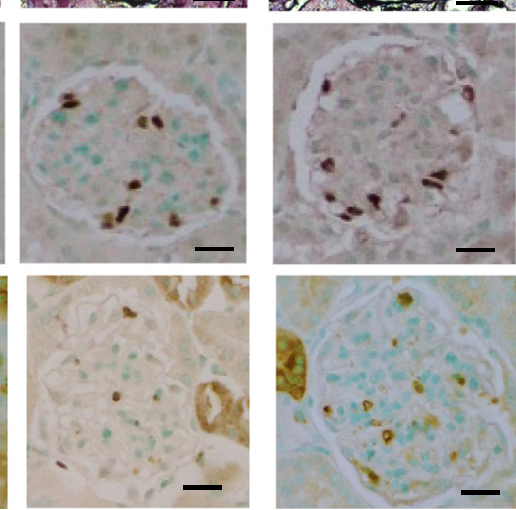

Silver

methenamine

Podocyte

p57

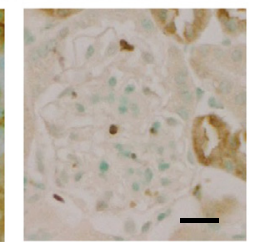

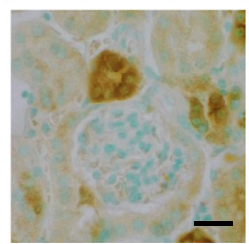

Figure 2 Inducible degrader of the low-density lipoprotein receptor (IDOL)-induced dyslipidemia augments albuminuria. Human degradation-resistant IDOL was expressed using a liver-specific adenoassociated virus (AAV) serotype DJ/8 [or enhanced green fluorescent protein (eGFP)-AAV-DJ/8 as a control] in 5-week-old male black and tan, brachyury (BTBR) wild-type (WT) and BTBR leptin-deficient (OB) mice. The mice were fed a high-fat diet for 18 weeks. A: Urine was collected during a 4- to 6-hour fasting period in the last week of the study, and albumin and creatinine were measured in the collected urine. B: At the end of the study, kidneys were sectioned and stained using periodic acid-Schiff (PAS), silver methenamine, podocyte p57, and macrophage-2 antigen (Mac-2) stains (both dark brown). C: The glomerulus area was measured and glomerular volume calculated. Data are expressed as means \pm SEM (A and C). $n=9$ to 15 (A and $\mathbf{C}) .{ }^{*} P<0.05,{ }^{* * *} P<0.001$ (two-way analysis of variance). Scale bars: $20 \mu \mathrm{m}$ (B).

did not alter obesity in WT or OB mice (Figure 1A). Furthermore, blood glucose levels were robustly elevated in both BTBR OB groups as early as 2 weeks (7 weeks of age) after initiation of diet (Figure 1B). Blood cholesterol levels were increased in leptin-deficient mice compared with WT mice, similar to previous reports, ${ }^{5}$ albeit worsened by the high-fat, cholesterol-containing diet (Figure 1C). Plasma cholesterol levels were further increased in BTBR OB mice after IDOL expression, consistent with IDOL-mediated degradation of the LDL receptor (Figure 1C). Similarly, plasma triglyceride levels were elevated by leptin deficiency and further increased by IDOL expression (Figure 1D). Expression of human IDOL resulted in an approximately twofold increase in total expression of MYLIP (the gene encoding IDOL) using primers that detect both human and mouse MYLIP (Figure 1E). Human-specific primers only detected MYLIP in human IDOL-AAV-injected mice (Supplemental Figure S1). Consistent with increased expression of MYLIP, liver LDL receptor levels were reduced (Figure $1 \mathrm{~F}$ ) in BTBR WT and BTBR OB mice receiving IDOL-AAV. Lipoprotein profiles demonstrated that leptin deficiency resulted in increased LDL-C compared with BTBR WT mice, and this was further increased by $30 \%$ in mice expressing IDOL (Figure 1, G and $\mathrm{H}$ ). Together, IDOL-induced dyslipidemia is consistent with reduced liver LDL receptor levels and is primarily driven by increased LDL-C.

IDOL-Induced Dyslipidemia Augments Albuminuria and Glomerular Macrophage Accumulation

Dyslipidemia has been proposed to be a risk factor for progression of DKD in individuals with T2D. ${ }^{13}$ To test whether IDOL-induced dyslipidemia would worsen DKD in BTBR OB mice, urine was collected during the last week of the study in fasting mice. Similar to a previous report, ${ }^{5}$ BTBR OB mice have significantly elevated albumin/creatinine ratios compared with WT mice (Figure 2A). This increase is driven by increased urinary albumin output because urinary creatinine levels were unchanged between the groups (Supplemental Figure S2A and Supplemental Table S1). IDOL-induced dyslipidemia in C57BL6 OB mice did not increase albuminuria above that of BTBR WT mice (Supplemental Figure S2B) and to the C57Bl/6 WT mice 
A

\section{Glomerular PAS}

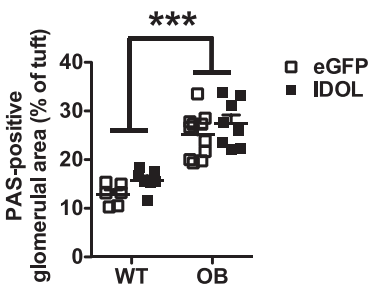

E

Podocyte density

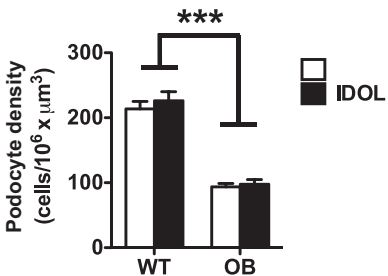

G

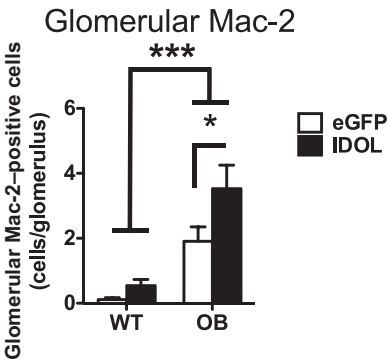

B

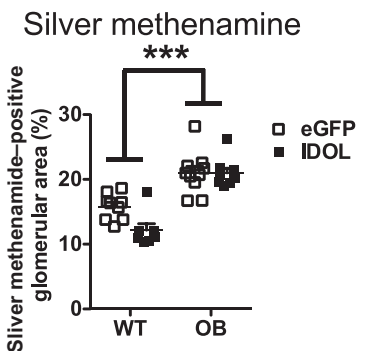

C
Cortex TG

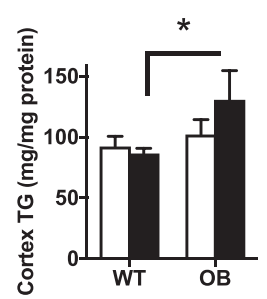

D

Glomerular Oil Red O
$\mathbf{F}$

\section{Glomerular ultrastructure}
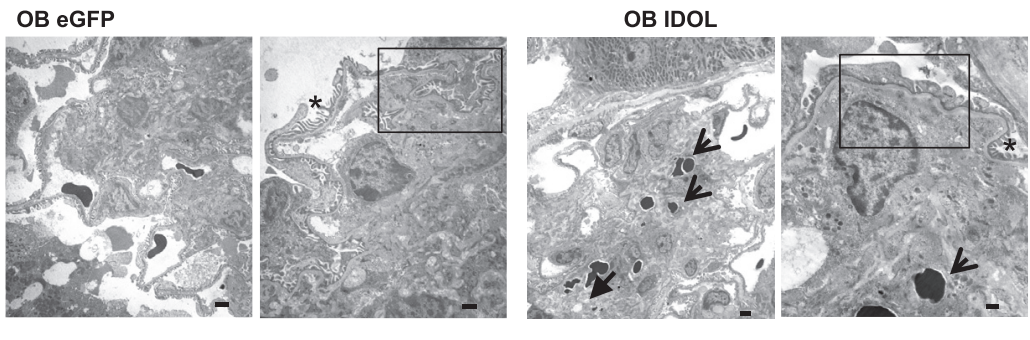

H

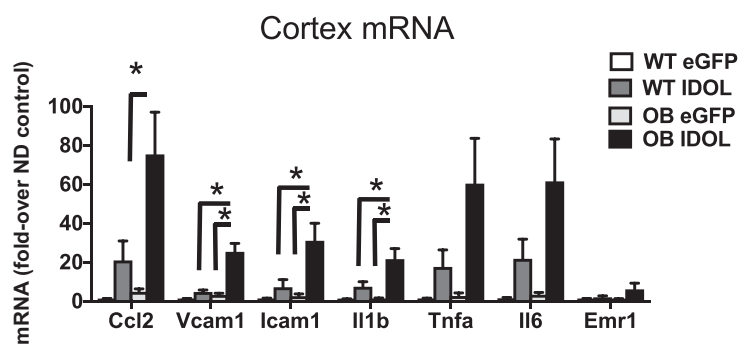

Figure 3 Inducible degrader of the low-density lipoprotein receptor (IDOL)-induced dyslipidemia results in increased kidney lipids and renal cortex inflammation and augments albuminuria. Human degradation-resistant IDOL was expressed using a liver-specific adenoassociated virus (AAV) serotype (DJ/8) [or enhanced green fluorescent protein (eGFP)-AAV-DJ/8 as a control] in 5-week-old male black and tan, brachyury (BTBR) wild-type (WT) and BTBR leptindeficient (OB) mice. The mice were fed a high-fat diet for 18 weeks. A and B: Mesangial expansion was estimated based on periodic acid-Schiff (PAS) (A) and silver methenamine stains (B) and expressed as percentage of total glomerular area. C: Triglycerides (TG) were measured in the cortex in a subset of animals and normalized to total protein amounts. D: Frozen sections were stained with 0il Red 0 and scored based on a 0 to 3 scoring system by investigators (F.K. and J.E.K.) blinded to the experimental groups. E: Podocyte numbers were measured and calculated as described in Materials and Methods. F: Electron micrographs of representative glomeruli that had expanded mesangial areas with matrix accumulation with lucencies that indicate mesangial lysis (solid arrow) and evidence of trapping of erythrocytes in the matrix (arrows). Boxed areas indicate effacement of podocyte foot processes, often adjacent to normal foot processes (asterisk). G: Glomerular macrophage-2 antigen (Mac-2) stain. H: RNA was extracted from the cortex and analyzed. Statistical analysis was performed on each gene separately (one-way analysis of variance). Data are expressed as means \pm SEM. $n=9$ to 15 (A-E and $\mathbf{G}) ; n=4$ (WT) and 8 (OB) (H). ${ }^{\star} P<0.05,{ }^{* *} P<0.001$ (two-way analysis of variance). Scale bars: $2 \mu \mathrm{m}, 100 \mathrm{~nm}, 2 \mu \mathrm{m}$, and $500 \mathrm{~nm}$ (F, left to right).

(data not shown). Interestingly, IDOL-induced dyslipidemia resulted in worsening of proteinuria, as assessed by albumin/ creatinine excretion, in BTBR OB mice (Figure 2A) but did not alter plasma cystatin $\mathrm{C}$ and blood urea nitrogen (Supplemental Figure S2, C and D).

Does IDOL-induced dyslipidemia worsen the histopathologic changes associated with DKD in BTBR OB mice? To address this question, kidneys were sectioned and stained for mesangial expansion using PAS and silver methenamine stains (Figure 2B). Glomerular hypertrophy was strikingly evident in both leptin-deficient groups, and IDOL-induced dyslipidemia did not alter glomerular hypertrophy (Figure 2, B and C). Furthermore, BTBR OB mice exhibit a distinct expansion of the mesangium, and this phenotype also was not altered by IDOL-induced dyslipidemia (Figures 2B and 3, A and B). Again, the histopathologic kidney changes observed in BTBR OB mice are specific to the BTBR strain of mice because the glomeruli in $\mathrm{C} 57 \mathrm{Bl} / 6 \mathrm{OB}$ mice were indistinguishable from those of BTBR WT mice (Supplemental Figure S2, E-I). Lipid deposition and altered triglyceride handling have been proposed to affect kidney disease. ${ }^{20,21}$ IDOL-induced dyslipidemia resulted in increased triglyceride accumulation in the renal cortex and increased Oil Red $\mathrm{O}$ staining in the glomerulus (Figure 3, C and D). The increased lipid accumulation in IDOL-expressing mice was observed in the 
A

En face atherosclerosis

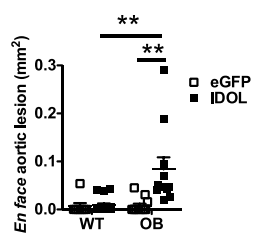

$\mathbf{B}$

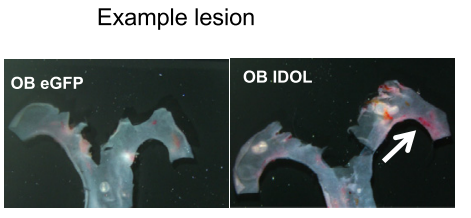

C

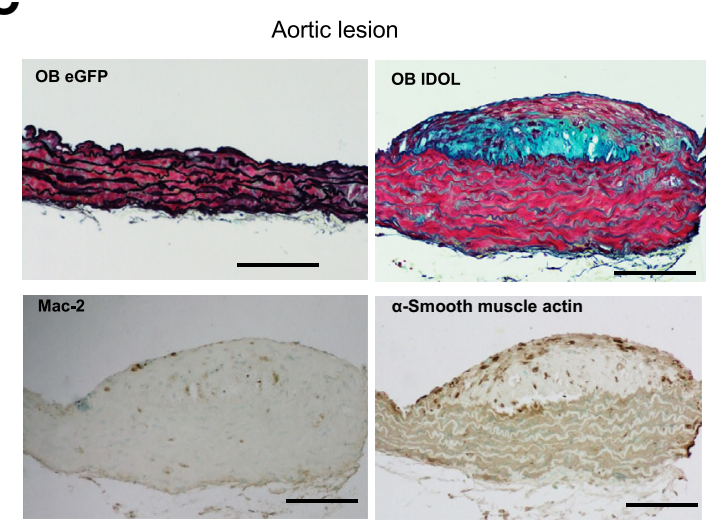

D

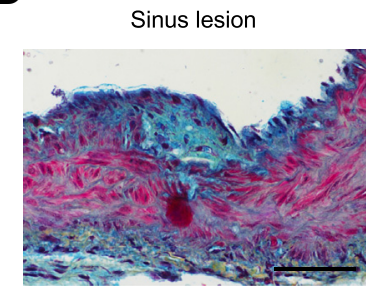

Figure 4 Inducible degrader of the lowdensity lipoprotein receptor (IDOL)-induced dyslipidemia results in early atherosclerotic lesion formation. Human degradation-resistant IDOL was expressed using a liver-specific adenoassociated virus (AAV) serotype DJ/8 [or enhanced green fluorescent protein (eGFP)-AAV-DJ/8 as a control] in 5-week-old male black and tan, brachyury (BTBR) wild-type (WT) and BTBR leptin-deficient $(O B)$ mice. The mice were fed a high-fat diet for 18 weeks. At the end of the study, aortas were dissected and opened longitudinally, and atherosclerosis was determined en face after Sudan IV staining (A). B: Examples of en face lesions in BTBR OB EGFP- and IDOL-injected mice (white arrow indicating lesion in aortic arch). C and D: Examples of cross-sectional lesions from that aorta (C) and aortic sinus (D). In C, the top panels show aortas stained with Movat's pentachrome stain. Note that only the IDOLinjected $\mathrm{OB}$ mouse's aorta has a lesion. The bottom panels show cross sections adjacent to the OB IDOL Movat's pentachrome-stained lesion (top right), stained for macrophage-2 antigen (Mac-2) and $\alpha$-smooth muscle actin, both resulting in a dark brown reaction product. Lesions could only be detected in IDOL-injected BTBR OB mice. Data are expressed as means $\pm \operatorname{SEM}(\mathbf{A}) . n=9$ to 15 (A). ${ }^{*} P<0.01$ (two-way analysis of variance). Scale bars: $100 \mu \mathrm{m}$. absence of changes in genes involved in fatty acid oxidation (Cptla, Acoxl, Ppara, and Ppargcla) in the cortex (data not shown).

Progression of DKD is also associated with injury and loss of podocytes in humans, ${ }^{22,23}$ and it has been previously found that BTBR OB mice have reduced podocyte numbers and density compared with WT mice. ${ }^{24}$ Consistently, it was observed that leptin deficiency in BTBR mice results in significantly fewer podocytes compared with BTBR WT mice (Figures 2B and 3E). However, IDOL-induced dyslipidemia did not alter measures of podocyte loss in BTBR WT or OB mice. Further ultrastructural analysis of the glomeruli indicated that leptin-deficient BTBR mice had a notable mesangial expansion with areas of mesangiolysis and trapping of erythrocytes in the expanding matrix (Figure 3F), consistent with a previous report. ${ }^{5}$ IDOL-induced dyslipidemia did not markedly alter those features. Furthermore, podocyte foot processes are focally effaced; however, this did not appear to be markedly increased in mice with IDOL-induced dyslipidemia (Figure 3F).

Glomerular macrophage accumulation has been proposed to be involved in kidney disease progression. ${ }^{25-28}$ To investigate glomerular macrophage accumulation in the BTBR mice, kidneys were stained for the macrophage marker Mac-2. Consistent with previous data, ${ }^{5,24}$ leptin deficiency resulted in more Mac-2-positive cells per glomerulus, and IDOL-induced dyslipidemia further exacerbated the number of Mac-2-positive cells that accumulated per glomeruli under OB conditions (Figure 3G).
Macrophage accumulation correlated with urinary albu$\mathrm{min} /$ creatinine ratio $\left(R^{2}=0.37, P<0.0001\right)$, potentially suggesting that the accelerated accumulation of macrophages may contribute to the increased albumin clearance. To further understand the increase in macrophage accumulation, mRNA from the renal cortex of all experimental groups was isolated and analyzed. Whereas leptin deficiency alone had no significant effect on inflammatory markers examined, the combination of leptin deficiency and IDOL-induced dyslipidemia markedly increased the expression of $\mathrm{Ccl} 2$, Vcam1, Icam1, and Il1b (Figure 3H). Together, these results suggest that IDOL-induced dyslipidemia worsens kidney function in diabetic BTBR OB mice through a process that may involve glomerular macrophage accumulation and local inflammation.

\section{IDOL-Induced Dyslipidemia Results in Early Atherosclerotic Lesion Formation}

At the end of the study, the extent of atherosclerosis was analyzed. Even though leptin deficiency in BTBR mice results in elevated cholesterol levels (approximately 400 $\mathrm{mg} / \mathrm{dL}$ ), BTBR OB mice injected with eGFP did not develop atherosclerosis during the 18-week study period, as detected by en face Sudan IV staining (Figure 4, A and B). Furthermore, IDOL did not result in atherosclerotic lesion formation in BTBR WT mice but resulted in formation of atherosclerotic lesions only in BTBR OB mice (Figure 4, A-D). To gain additional insight into the effects of increased plasma LDL-C by IDOL on atherosclerotic lesion 
morphologic features, the aorta and the aortic sinus were sectioned and stained with Movat's pentachrome stain. Only BTBR OB animals with IDOL-induced dyslipidemia had detectable lesions in the aorta or the aortic sinus (Figure 4, C and $\mathrm{D})$. The atherosclerotic lesions were generally small, especially in the aortic sinus, but lesions with a smooth muscle-containing fibrous cap could be detected in the aorta of BTBR OB mice with IDOL-induced dyslipidemia (Figure 4D). These lesions also contained Mac-2-positive macrophages (Figure 4C). No lesions could be detected in any other group.

\section{IDOL-Induced Dyslipidemia Results in Atherosclerosis in $\mathrm{C} 57 \mathrm{Bl} / 6$ Mice}

C57B1/6 mice are prone to development of atherosclerosis if atherosclerotic dyslipidemia is induced, for example, by deleting the LDL receptor. ${ }^{29}$ To investigate the effect of IDOL-AAV-DJ/8 on atherosclerosis in C57Bl/6 mice, WT and $\mathrm{OB}$ C57B1/6 mice were injected with IDOL AAV and were then fed the high-fat, cholesterol-containing diet for 17 weeks. Similar to the BTBR mice, leptin deficiency resulted in marked weight gain (Supplemental Figure S3A) and hypercholesterolemia (Supplemental Figure S3B). In contrast to BTBR mice, leptin deficiency did not result in hyperglycemia in C57B1/6 mice (Supplemental Figure S3C). IDOL-induced dyslipidemia caused significant en face atherosclerosis in aortas of the $\mathrm{C} 57 \mathrm{Bl} / 6 \mathrm{OB}$ mice, as well as clearly detectible lesions in the aortic sinus (Supplemental Figure S3, D-F), reinforcing that IDOL-AAV-DJ/8 can be used as a tool to induce atherosclerosis and that $\mathrm{C} 57 \mathrm{Bl} / 6$ is an atherosclerosis-susceptible genetic background.

\section{Discussion}

In the current study, we found the feasibility of using an AAV approach to target degradation of the LDL receptor in the BTBR mouse strain and thus render them susceptible to atherosclerosis. This approach circumvents the timeconsuming breeding that is commonly used to introduce proatherogenic dyslipidemia in mice and is adaptable to many different strains of mice. This is particularly important in the BTBR OB strain because this strain is susceptible to microvascular diabetes complications but does not develop significant atherosclerosis. Thus, the BTBR strain is known for its susceptibility to glucose dysregulation, ${ }^{30}$ and with the deletion of the leptin gene, the BTBR OB mouse has emerged as one of the models that best mimics human DKD. ${ }^{5,24,31}$ Furthermore, BTBR OB mice have signs of other microvascular complications, such as neuropathy and retinopathy, but are not hypertensive. ${ }^{5,32,33}$ However, no atherosclerosis can be detected in BTBR OB mice without increasing LDL by reducing its clearance. The IDOL-AAV-DJ/8 approach rapidly generates a T2D mouse model of combined microvascular and macrovascular diabetes complications. Although the relative level of reduction of the LDL receptor could be improved on, this study clearly suggests that BTBR mice can be used to study atherosclerosis. Another strategy that successfully circumvented breeding onto proatherogenic backgrounds is overexpression of proprotein convertase subtilisin kexin 9 (PCSK9), which also results in reduced LDL receptor levels. ${ }^{34}$ Both PCSK9 and IDOL result in elevated circulating levels of plasma cholesterol, with increases in primarily non-HDL-C. Overexpression of IDOL has previously been shown to elevate non-HDL-C levels. ${ }^{9,12}$ IDOL overexpression using AAVs is another molecular tool in a scientist's toolbox to generate models of atherosclerosis.

Our study also reinforces that the commonly used C57B1/ 6 strain for atherosclerosis is more susceptible to the development of atherosclerosis than the BTBR strain because these animals had larger and more advanced lesions than the BTBR mice.

Elevated glucose levels associated with diabetes have been proposed to be a major underlying mechanism of macrovascular and microvascular complications. ${ }^{35}$ Clinical trials and epidemiologic studies clearly support the importance of suboptimal blood glucose control in development of microvascular diabetes complications ${ }^{36}$; however, lipids have been suggested to play a pathologic role also in the progression of kidney disease. ${ }^{37}$ Our results support this conclusion because IDOL expression resulted in worsened albuminuria in BTBR OB mice. A small meta-analysis and a secondary analysis from cardiovascular end point studies supports this finding. ${ }^{38,39}$ Furthermore, Kang et al ${ }^{21}$ recently found that altered fatty acid metabolism is strongly correlated with progression to fibrotic renal disease. Falkevall et $\mathrm{a}^{40}$ suggested that glomerular lipid deposition correlated with DKD. However, the larger Study of Heart and Renal Protection trial puts this into question because no beneficial effects on end-stage renal disease or estimated glomerular filtration rates were observed using statins to lower lipids. ${ }^{41,42}$ A recent retrospective study ${ }^{13}$ that found that specific components of diabetic dyslipidemia (ie, low HDL-C and high triglyceride levels) were independent risk factors for progression of renal disease in individuals with T2D. Our data suggest that diabetic dyslipidemia indeed accelerates, at least some aspects, of DKD. Thus, IDOLinduced dyslipidemia results in increased urinary albumin secretion and was associated with increased macrophage accumulation and inflammation in glomeruli. This finding suggests that dyslipidemia might primarily accelerate the inflammatory component of DKD, which may be the mechanism that results in increased albuminuria in IDOL-induced dyslipidemia. Macrophages are known to accumulate in glomeruli and the interstitium in DKD and are present early in the disease pathogenesis. Macrophage chemokines, such as chemokine (C-C motif) ligand (CCL)2, are known to be increased in diabetic kidneys and consistent with up-regulation of CCL-2. Macrophage numbers are also increased and correlate with disease stage 
in mouse models and humans with diabetes. ${ }^{26,28,43}$ Consistently, preventing macrophage accumulation in glomeruli using CCL-2-deficient mice or, more recently, pharmacologic inhibition of CCR2 in humans improves urine albumin secretion under T2D conditions, implying a pathologic role of CCL-2-mediated macrophage accumulation in DKD. ${ }^{27,44-46} \mathrm{~A}$ testable hypothesis that emerges from the present study is that lipids stimulate macrophage accumulation in the glomerulus in DKD, similar to what happens in the artery wall, and that the underlying mechanisms are similar.

In summary, by inducing hepatic degradation of the LDL receptor, we generated a T2D model of combined DKD and atherosclerosis. This model provides a new tool to study mechanisms, interactions, and treatment strategies of kidney disease and atherosclerosis in T2D.

\section{Acknowledgments}

J.E.K. is the guarantor of this work and, as such, had full access to all the data in the study and takes responsibility for the integrity of the data and the accuracy of the data analysis in the manuscript; all authors reviewed the manuscript and provided final approval for submission.

\section{Supplemental Data}

Supplemental material for this article can be found at https://doi.org/10.1016/j.ajpath.2017.10.012.

\section{References}

1. Writing Group Members, Mozaffarian D, Benjamin EJ, Go AS, Arnett DK, Blaha MJ, et al: Executive summary: heart disease and stroke statistics-2016 update: a report from the American Heart Association. Circulation 2016, 133:447-454

2. de Boer IH, Rue TC, Hall YN, Heagerty PJ, Weiss NS, Himmelfarb J: Temporal trends in the prevalence of diabetic kidney disease in the United States. JAMA 2011, 305:2532-2539

3. Afkarian M, Sachs MC, Kestenbaum B, Hirsch IB, Tuttle KR, Himmelfarb J, de Boer IH: Kidney disease and increased mortality risk in type 2 diabetes. J Am Soc Nephrol 2013, 24:302-308

4. Fox CS, Matsushita K, Woodward M, Bilo HJ, Chalmers J, Heerspink HJ, Lee BJ, Perkins RM, Rossing P, Sairenchi T, Tonelli M, Vassalotti JA, Yamagishi K, Coresh J, de Jong PE, Wen CP, Nelson RG; Chronic Kidney Disease Prognosis Consortium: Associations of kidney disease measures with mortality and end-stage renal disease in individuals with and without diabetes: a meta-analysis. Lancet 2012, 380:1662-1673

5. Hudkins KL, Pichaiwong W, Wietecha T, Kowalewska J, Banas MC, Spencer MW, Muhlfeld A, Koelling M, Pippin JW, Shankland SJ, Askari B, Rabaglia ME, Keller MP, Attie AD, Alpers CE: BTBR $\mathrm{Ob} / \mathrm{Ob}$ mutant mice model progressive diabetic nephropathy. J Am Soc Nephrol 2010, 21:1533-1542

6. Ishibashi S, Brown MS, Goldstein JL, Gerard RD, Hammer RE, Herz J: Hypercholesterolemia in low density lipoprotein receptor knockout mice and its reversal by adenovirus-mediated gene delivery. J Clin Invest 1993, 92:883-893
7. Ishibashi S, Goldstein JL, Brown MS, Herz J, Burns DK: Massive xanthomatosis and atherosclerosis in cholesterol-fed low density lipoprotein receptor-negative mice. J Clin Invest 1994, 93:1885-1893

8. Ishibashi S, Herz J, Maeda N, Goldstein JL, Brown MS: The tworeceptor model of lipoprotein clearance: tests of the hypothesis in "knockout" mice lacking the low density lipoprotein receptor, apolipoprotein E, or both proteins. Proc Natl Acad Sci U S A 1994, 91: $4431-4435$

9. Zelcer N, Hong C, Boyadjian R, Tontonoz P: LXR regulates cholesterol uptake through Idol-dependent ubiquitination of the LDL receptor. Science 2009, 325:100-104

10. Calkin AC, Goult BT, Zhang L, Fairall L, Hong C, Schwabe JW, Tontonoz P: FERM-dependent E3 ligase recognition is a conserved mechanism for targeted degradation of lipoprotein receptors. Proc Natl Acad Sci U S A 2011, 108:20107-20112

11. Ibrahim S, Somanathan S, Billheimer J, Wilson JM, Rader DJ: Stable liver-specific expression of human IDOL in humanized mice raises plasma cholesterol. Cardiovasc Res 2016, 110:23-29

12. Calkin AC, Lee SD, Kim J, Van Stijn CM, Wu XH, Lusis AJ, Hong C, Tangirala RI, Tontonoz P: Transgenic expression of dominant-active IDOL in liver causes diet-induced hypercholesterolemia and atherosclerosis in mice. Circ Res 2014, 115:442-449

13. Russo GT, De Cosmo S, Viazzi F, Pacilli A, Ceriello A, Genovese S, Guida P, Giorda C, Cucinotta D, Pontremoli R, Fioretto P; AMD-Annals Study Group: Plasma triglycerides and HDL-C levels predict the development of diabetic kidney disease in subjects with type 2 diabetes: the AMD annals initiative. Diabetes Care 2016, 39:2278-2287

14. Grimm D, Lee JS, Wang L, Desai T, Akache B, Storm TA, Kay MA: In vitro and in vivo gene therapy vector evolution via multispecies interbreeding and retargeting of adeno-associated viruses. J Virol 2008, 82:5887-5911

15. Renard CB, Kramer F, Johansson F, Lamharzi N, Tannock LR, von Herrath MG, Chait A, Bornfeldt KE: Diabetes and diabetes-associated lipid abnormalities have distinct effects on initiation and progression of atherosclerotic lesions. J Clin Invest 2004, 114:659-668

16. Lewis KE, Kirk EA, McDonald TO, Wang S, Wight TN, O’Brien KD, Chait A: Increase in serum amyloid a evoked by dietary cholesterol is associated with increased atherosclerosis in mice. Circulation 2004, 110:540-545

17. Vallerie SN, Kramer F, Barnhart S, Kanter JE, Breyer RM, Andreasson KI, Bornfeldt KE: Myeloid cell prostaglandin E2 receptor EP4 modulates cytokine production but not atherogenesis in a mouse model of type 1 diabetes. PLoS One 2016, 11:e0158316

18. Venkatareddy M, Wang S, Yang Y, Patel S, Wickman L, Nishizono R, Chowdhury M, Hodgin J, Wiggins PA, Wiggins RC: Estimating podocyte number and density using a single histologic section. J Am Soc Nephrol 2014, 25:1118-1129

19. Kanter JE, Kramer F, Barnhart S, Averill MM, Vivekanandan-Giri A, Vickery T, Li LO, Becker L, Yuan W, Chait A, Braun KR, PotterPerigo S, Sanda S, Wight TN, Pennathur S, Serhan CN, Heinecke JW, Coleman RA, Bornfeldt KE: Diabetes promotes an inflammatory macrophage phenotype and atherosclerosis through acyl-CoA synthetase 1. Proc Natl Acad Sci U S A 2012, 109:E715-E724

20. Herman-Edelstein M, Scherzer P, Tobar A, Levi M, Gafter U: Altered renal lipid metabolism and renal lipid accumulation in human diabetic nephropathy. J Lipid Res 2014, 55:561-572

21. Kang HM, Ahn SH, Choi P, Ko YA, Han SH, Chinga F, Park AS, Tao J, Sharma K, Pullman J, Bottinger EP, Goldberg IJ, Susztak K: Defective fatty acid oxidation in renal tubular epithelial cells has a key role in kidney fibrosis development. Nat Med 2015, 21:37-46

22. Reidy K, Kang HM, Hostetter T, Susztak K: Molecular mechanisms of diabetic kidney disease. J Clin Invest 2014, 124:2333-2340

23. Andeen NK, Nguyen TQ, Steegh F, Hudkins KL, Najafian B, Alpers CE: The phenotypes of podocytes and parietal epithelial cells may overlap in diabetic nephropathy. Kidney Int 2015, 88:1099-1107

24. Pichaiwong W, Hudkins KL, Wietecha T, Nguyen TQ, Tachaudomdach C, Li W, Askari B, Kobayashi T, O'Brien KD, 
Pippin JW, Shankland SJ, Alpers CE: Reversibility of structural and functional damage in a model of advanced diabetic nephropathy. J Am Soc Nephrol 2013, 24:1088-1102

25. Galkina E, Ley K: Leukocyte recruitment and vascular injury in diabetic nephropathy. J Am Soc Nephrol 2006, 17:368-377

26. Chow F, Ozols E, Nikolic-Paterson DJ, Atkins RC, Tesch GH: Macrophages in mouse type 2 diabetic nephropathy: correlation with diabetic state and progressive renal injury. Kidney Int 2004, 65:116-128

27. Chow FY, Nikolic-Paterson DJ, Ozols E, Atkins RC, Rollin BJ, Tesch GH: Monocyte chemoattractant protein-1 promotes the development of diabetic renal injury in streptozotocin-treated mice. Kidney Int 2006, 69:73-80

28. Nguyen D, Ping F, Mu W, Hill P, Atkins RC, Chadban SJ: Macrophage accumulation in human progressive diabetic nephropathy. Nephrology (Carlton) 2006, 11:226-231

29. Paigen B, Ishida BY, Verstuyft J, Winters RB, Albee D: Atherosclerosis susceptibility differences among progenitors of recombinant inbred strains of mice. Arteriosclerosis 1990, 10:316-323

30. Stoehr JP, Nadler ST, Schueler KL, Rabaglia ME, Yandell BS, Metz SA, Attie AD: Genetic obesity unmasks nonlinear interactions between murine type 2 diabetes susceptibility loci. Diabetes 2000, 49: 1946-1954

31. Alpers CE, Hudkins KL: Mouse models of diabetic nephropathy. Curr Opin Nephrol Hypertens 2011, 20:278-284

32. O'Brien PD, Hur J, Hayes JM, Backus C, Sakowski SA, Feldman EL: BTBR ob/ob mice as a novel diabetic neuropathy model: neurological characterization and gene expression analyses. Neurobiol Dis 2015, 73:348-355

33. Zhi Z, Chao JR, Wietecha T, Hudkins KL, Alpers CE, Wang RK: Noninvasive imaging of retinal morphology and microvasculature in obese mice using optical coherence tomography and optical microangiography. Invest Ophthalmol Vis Sci 2014, 55:1024-1030

34. Bjorklund MM, Hollensen AK, Hagensen MK, Dagnaes-Hansen F, Christoffersen C, Mikkelsen JG, Bentzon JF: Induction of atherosclerosis in mice and hamsters without germline genetic engineering. Circ Res 2014, 114:1684-1689

35. Brownlee M: The pathobiology of diabetic complications: a unifying mechanism. Diabetes 2005, 54:1615-1625

36. Nathan DM, Bayless M, Cleary P, Genuth S, Gubitosi-Klug R, Lachin JM, Lorenzi G, Zinman B, Group DER: Diabetes control and complications trial/epidemiology of diabetes interventions and complications study at 30 years: advances and contributions. Diabetes 2013, 62:3976-3986

37. Moorhead JF, Chan MK, El-Nahas M, Varghese Z: Lipid nephrotoxicity in chronic progressive glomerular and tubulo-interstitial disease. Lancet 1982, 2:1309-1311

38. Fried LF, Orchard TJ, Kasiske BL: Effect of lipid reduction on the progression of renal disease: a meta-analysis. Kidney Int 2001, 59:260-269

39. Ritz E, Wanner C: Lipid changes and statins in chronic renal insufficiency. J Am Soc Nephrol 2006, 17:S226-S230

40. Falkevall A, Mehlem A, Palombo I, Heller Sahlgren B, Ebarasi L, He L, Ytterberg AJ, Olauson H, Axelsson J, Sundelin B, Patrakka J, Scotney P, Nash A, Eriksson U: Reducing VEGF-B signaling ameliorates renal lipotoxicity and protects against diabetic kidney disease. Cell Metab 2017, 25:713-726

41. Baigent C, Landray MJ, Reith C, Emberson J, Wheeler DC, Tomson C, et al: The effects of lowering LDL cholesterol with simvastatin plus ezetimibe in patients with chronic kidney disease (Study of Heart and Renal Protection): a randomised placebo-controlled trial. Lancet 2011, 377:2181-2192

42. Haynes R, Lewis D, Emberson J, Reith C, Agodoa L, Cass A, Craig JC, de Zeeuw D, Feldt-Rasmussen B, Fellstrom B, Levin A, Wheeler DC, Walker R, Herrington WG, Baigent C, Landray MJ, Group SC, Group SC: Effects of lowering LDL cholesterol on progression of kidney disease. J Am Soc Nephrol 2014, 25:1825-1833

43. Chow FY, Nikolic-Paterson DJ, Atkins RC, Tesch GH: Macrophages in streptozotocin-induced diabetic nephropathy: potential role in renal fibrosis. Nephrol Dial Transplant 2004, 19:2987-2996

44. Seok SJ, Lee ES, Kim GT, Hyun M, Lee JH, Chen S, Choi R, Kim HM, Lee EY, Chung CH: Blockade of CCL2/CCR2 signalling ameliorates diabetic nephropathy in $\mathrm{db} / \mathrm{db}$ mice. Nephrol Dial Transplant 2013, 28:1700-1710

45. de Zeeuw D, Bekker P, Henkel E, Hasslacher C, Gouni-Berthold I, Mehling H, Potarca A, Tesar V, Heerspink HJ, Schall TJ; CCX140-B Diabetic Nephropathy Study Group: The effect of CCR2 inhibitor CCX140-B on residual albuminuria in patients with type 2 diabetes and nephropathy: a randomised trial. Lancet Diabetes Endocrinol 2015, 3:687-696

46. Perez-Gomez MV, Sanchez-Nino MD, Sanz AB, Zheng B, MartinCleary C, Ruiz-Ortega M, Ortiz A, Fernandez-Fernandez B: Targeting inflammation in diabetic kidney disease: early clinical trials. Expert Opin Investig Drugs 2016, 25:1045-1058 